\title{
Metacognitive-Focused Occupational Therapy for Substance Abuse in Medical-Social Service Collaboration: a Case Report
}

\author{
KCK Lam, EML Fung, HF Chan, FTM Louie, F Chan
}

\begin{abstract}
We describe a 26-year-old man who underwent Metacognitive-Focused Occupational Therapy for his substance abuse problems.
\end{abstract}

Key words: Metacognition; Occupational therapy; Substance-related disorders

Kino CK Lam, Occupational Therapy Department, Alice Ho Miu Ling Nethersole Hospital, Hong Kong

Ernest ML Fung, Occupational Therapy Department, Alice Ho Miu Ling Nethersole Hospital, Hong Kong

Hiu Fai Chan, Cheer Lutheran Centre (Counselling Centre for Psychotropic Substance Abusers)

Frances TM Louie, Occupational Therapy Department, Alice Ho Miu Ling Nethersole Hospital, Hong Kong

F Chan, MBChB, MRCPsych, FHKCPsych, FHKAM (Psychiatry), Department of Psychiatry, North District Hospital, Hong Kong

Address for correspondence: Kino CK Lam, Occupational Therapy Department, Alice Ho Miu Ling Nethersole Hospital, Hong Kong.

Email: ikino28@gmail.com

Submitted: 5 February 2020; Accepted: 9 November 2020

\section{Background}

People with substance abuse disorders are often reluctant to attend psychiatric follow-up or rehabilitation programmes. To enhance treatment compliance, a medical-social service collaboration model has been adopted in Hong Kong. The goals are to help substance abusers to resist cravings and to re-engage meaningful daytime occupations/activities. Metacognitive-Focused Occupational Therapy (MFOT) is a specialised rehabilitation programme that incorporates advanced psychotherapeutic techniques (ie, metacognitive therapy) into occupational lifestyle redesign conducted by experienced occupational therapists in counselling centres for psychotropic substance abusers. These centres support patients to attend substance abuse clinics under the Hospital Authority for early psychiatric treatment and rehabilitation.

\section{Case Presentation}

In August 2017, a 26-year-old man was diagnosed as having drug-induced psychosis. Since age 16 years, he had repeatedly engaged in substance abuse when he felt stress at work or with relationship problems. He spent much time in ruminative thinking on his perceived failures and worried whether he would be able to cease substance abuse and improve family relationships. He monitored his mood status and found that he always fell into a depressed mood. This 'threat-monitoring' further reinforced his negative belief of 'I have no hope'. He tried to quit substance abuse but failed repeatedly. The longest abstinence period was only 6 months. He approached a counselling centre for psychotropic substance abusers for treatment.

The first stage of MFOT was to raise the patient's metacognitive awareness on cognitive attentional syndrome, based on the A-M-C formulation of internal antecedent, meta-beliefs and cognitive attentional syndrome, and emotional consequences (Figure). Guided discovery on the current emotions, cognitive symptoms, and behavioural changes was conducted first. The trigger thoughts were then explored with meta-level questioning to elicit the patient's cognitive attentional syndrome and his underlying metacognitive beliefs. He was introduced to the idea that substance abuse was an unhelpful coping behaviour to deal with negative thoughts and emotions. Mal-adaptive metacognitive beliefs were the underlying driving force of cognitive attentional syndrome, which typically involves usefulness of some thinking strategies and substancetaking behaviour and uncontrollability on thinking. The improper mode of processing to intrusive urge or thought to take substance would re-activate the cognitive attentional syndrome and associated metacognitive beliefs. To create a new mode of processing to internal triggers, detached mindfulness was taught to the patient to observe inner events such as negative thoughts and urges without trying to control or change them as a 'do-nothing' strategy before postponement of extended thinking and challenging the meta-beliefs. When the patient completed the programme, the cognitive attentional syndrome was eliminated, and his attentional resources were spent outward on meaningful leisure and work-related activities.

The patient showed $65 \%$ improvement in selfefficacy to avoid drug use. Since 2017, he has successfully ceased substance abuse behaviour. He reported being more capable to control his thinking (a sign of improvement in negative meta-belief) and did not engage much in extended thinking (a sign of improvement in positive meta-belief). He gave up all mal-adaptive coping behaviours such as social avoidance in response to negative mood. His life role has been reformed to a productive worker with stable job record and mental condition. 


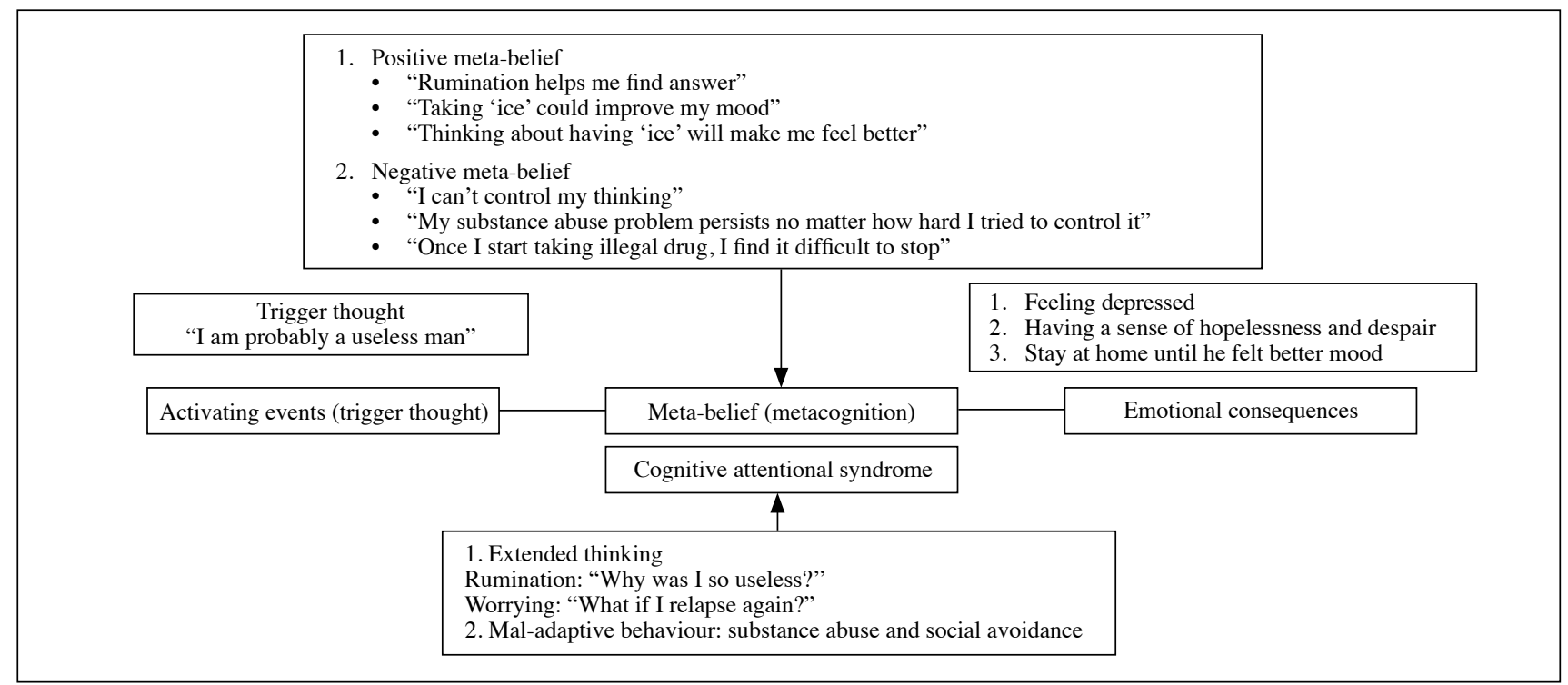

Figure. Overview of patient response in A-M-C formulation of internal antecedent, meta-beliefs and cognitive attentional syndrome, and emotional consequences

\section{Discussion}

Metacognitive therapy is a third-wave psychotherapy for emotional disorders. It aims to eliminate a problematic pattern of thinking and cognitive processing called the cognitive attentional syndrome, which consists of worry and rumination (extended thinking), fixated attention to threat, and unhelpful self-regulatory strategies or coping behaviours. Metacognitive therapy targets the interruption of the erroneous belief on cognition and the unhelpful thinking process. ${ }^{1}$ It is superior to traditional content-focused therapy in terms of short- and long-term outcomes in treating general anxiety disorder. ${ }^{1}$ Abusers of psychoactive substances have difficulty resisting cravings and often prioritise drug use over other daily and healthy activities. Metacognitive therapy has been used to target cognitive attentional syndrome and metacognitive beliefs in addictive behaviour. ${ }^{2-6}$ Cognitive attentional syndrome affects deliberate volition and the self-regulation system that plays a core role in restraining or maintaining the addictive behaviours. Substance abuse behaviour is a mal-adaptive coping strategy to negative thoughts/affect; removal of cognitive attentional syndrome style of thinking is beneficial and prerequisite in treatment. MFOT uses metacognitive therapy techniques to resolve the cognitive attentional syndrome in the process of ceasing substance abuse.

MFOT typically includes one intake session and four to six therapy sessions, one per week. It is more suitable for those with comorbid mental disorders such as depression, anxiety, and obsessive-compulsive disorder, as cognitive attentional syndrome is highly related to addictive behaviours that act to cope with the negative affect. MFOT enhances the self-regulatory executive function to resist craving by eliminating the cognitive attentional syndrome. It integrates occupational lifestyle redesign targeting the life-role reforming and functional enhancement in four life domains such as re-engaging in work life and rebuilding a healthy social circle. Participants are expected to decrease their frequency of drug use, improve the self-efficacy to avoid drug use, and enhance the occupational life functioning. In the intake session, assessment is conducted to find out which life domain the participant wants to improve. The discrepancy between the satisfaction level and the needs level is a key to elicit motivation to seek improvement. Regular discussion and implementation on the action plan in following sessions help participants acquire tendencies to automatically response and perform in consistent ways to reach the desired life goal.

\section{Conclusion}

Treating people with substance abuse problems needs a multidisciplinary intervention and medical-social service collaboration. MFOT that incorporates metacognitive therapy and occupational therapy is an effective programme for treating substance abuse.

\section{References}

1. Nordahl HM, Borkovec TD, Hagen R, Kennair LEO, Hjemdal O, Solem S, et al. Metacognitive therapy versus cognitive-behavioural therapy in adults with generalised anxiety disorder. BJPsych Open 2018;4:393-400. Crossref

2. Spada MM, Caselli G, Nikčević AV, Wells A. Metacognition in addictive behaviors. Addict Behav 2015;44:9-15. Crossref

3. Wells A. Emotional Disorders and Metacognition: Innovative Cognitive Therapy. Chichester, UK: Wiley; 2000.

4. Wells A. Metacognitive Therapy for Anxiety and Depression. New York: Guilford Press; 2009.

5. Wells A. Advances in metacognitive therapy. Int $J$ Cogn Ther 2013;6:186-201. Crossref

6. Wells A, Matthews G. Modelling cognition in emotional disorder: the S-REF model. Behav Res Ther 1996;34:881-8. Crossref 